\title{
DEVELOPMENT AND VALIDATION OF STRUCTURAL MODELS OF HUMAN POSTURE
}

\author{
R. BARAUSKAS and R. KRUŠINSKIENE \\ Faculty of Informatics, Kaunas University of Technology \\ Studentus str. 50, LT-51368 Kaunas, Lithuania \\ E-mail: rimantas.barauskas@ktu.lt; r.krusinskiene@lkka.lt
}

Received September 1, 2005; revised February 7, 2006

\begin{abstract}
A time law of small vibrations of the center of pressure (COP) of humans in standing position (human posture) provides a useful information about the physical and health condition of an individual. In this work controlled inverted pendulum (CIP) model with a single degree of freedom (DOF) is used in order to simulate the vibration of COP of a human body in anterio-posteriori (forwardbackward) direction during still standing. A method for identification of the CIP model parameters is based on numerical computation of the sensitivities of the penalty-type error function to small variations of model parameters. The approach is applicable to structural models with any number of DOF and any structural complexity. Numerical example demonstrates the model parameter identification results providing nearly the best match of the model behaviour to the real experimental record of COP position.
\end{abstract}

Key words: controlled inverted pendulum, human posture, optimal control, sensitivity functions

\section{Introduction}

The time law of small vibrations of the center of pressure (COP) of humans in standing position (human posture) may provide useful information about the physical and health condition of an individual. One of the most popular ways to measure standing stability is to register movements of COP on the base of support. The resulting figure is called a stabilogram. COP signal represents a collective outcome of all systems that are responsible for maintaining body upright.

Different models are used in order to explain this, at the first glance, quite chaotic signal (see Fig. 1): pinned polymer [?], auto regressive [?] or fuzzy logic [?] models. Nevertheless most of them fail to identify the vibration law of COP 


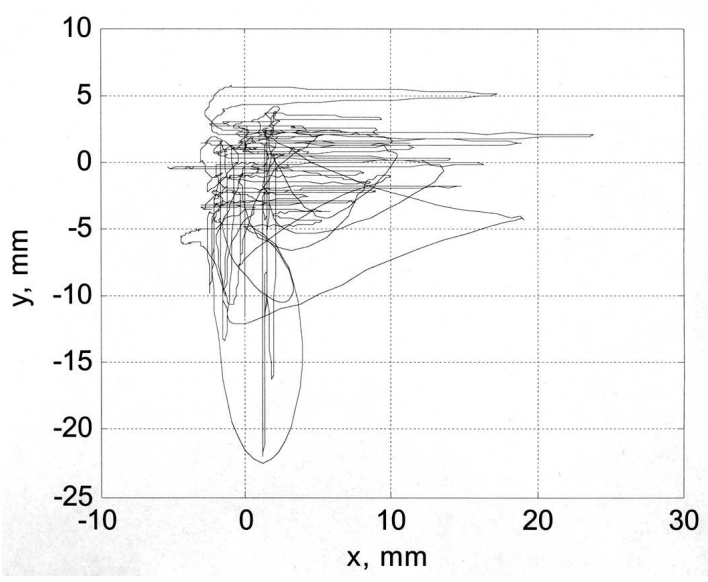

Figure 1. Movement of COP on a base of support.

in physiologically meaningful terms and are not very popular in the clinical context so far. One of the most popular models is the controlled inverted pendulum (CIP) model which represents movements of COP in anterio-posteriori (forward-backward) direction during still standing.

G. Morasso and M.Schieppati in [?] related control of the inverted pendulum with two control mechanisms: open-loop control mechanism used over short-term period and closed-loop control mechanism used over long-term period. While R. Peterka in [?] demonstrated that the modeling results consistent with experimental data may be obtained using only one closed-loop control mechanism for both - long and short term - periods. In both cases CIP model parameters were selected empirically by comparing the computed and experimental data [?, ?, ?].

The formal method for CIP model and its' closed-loop control parameters identification is presented in [?]. The method employs the optimum control technique [?]. This paper is an extended version of [?]. It presents the proof of the general case of the control parameters identification method.

\section{Methods}

\subsection{Controlled inverted pendulum model and error function formulation}

In CIP model (Fig. 2) human body is represented as a rigid body with the center of mass (COM) oscillating around the ankle joint. The dynamic equation of such a system is obtained on the base of the angular momentum principle by making assumptions that the posture deviates away from upright position due to some physiological factors (e.g. breathing), as well as due to the torque generated by the gravity force. The influence of such factors in the CIP model is represented as the cumulative disturbance torque $T_{d}(t)$. 


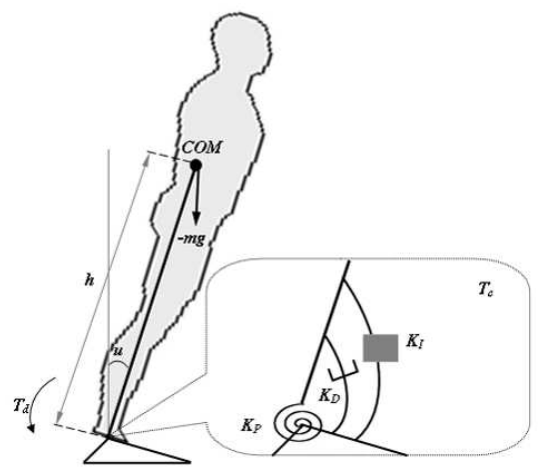

Figure 2. Controlled Inverted Pendulum (CIP) model of a human posture.

According to [?] the cumulative disturbance torque may be represented as low-filtered Gaussian noise. In order to counteract the disturbance torque human body produces the corrective torque $T_{c}(t)$. The corrective torque is assumed to be a linear function of the sway angle, angular velocity and the time integral of the sway angle. In [?] it has been referred to as proportional, integral and differential (PID) controller. Finally the single DOF CIP model is described as

$$
I \ddot{u}(t)-m g h u(t)=T_{d}(t)-T_{c}(t),
$$

where: $m$ is body mass, $I$ mass moment of inertia of the body about the ankle joint, $h$ distance of COM from the ankle joint, $u$ sway angle, $g$ gravitational acceleration, $T_{d}$ cumulative disturbance torque obtained from the equation

$$
T_{d}(t)+B \dot{T}_{d}(t)=A x(t),
$$

$x(t)$ Gaussian noise, $A, B$ first order low-filter coefficients,

$$
T_{c}(t)=K_{P} u(t)+K_{I} \int_{t} u(t) d t+K_{D} \dot{u}(t)
$$

is corrective torque, $K_{P}, K_{I}, K_{D}$ are coefficients.

Proper values of CIP model parameters $K_{P}, K_{I}, K_{D}, A$ and $B$ have to be determined in order to make the response of the model close to time law of vibrations of COP of a real human body. The quantitative measure of the deviation of the model behavior from the available experimental record is introduced by means of the chosen error function as

$$
\begin{aligned}
& J(u)=\int_{0}^{T} \psi(u, \dot{u}, \ddot{u}) d t, \\
& \psi(u, \dot{u}, \ddot{u})=\frac{1}{2}\left(u_{C O P}(t)-u_{r e f}(t)\right)^{2},
\end{aligned}
$$


where $u_{\text {ref }}$ are $\mathrm{COP}$ values recorded during experiment, $u_{C O P}$ are COP values calculated from CIP model data according to the formula presented in [?]:

$$
u_{C O P}(t)=h u(t)-\frac{I \ddot{u}(t)}{m g} .
$$

\subsection{Sensitivity coefficients of the error function}

\subsubsection{The method}

Consider an elastic structure presented by the dynamic equation as

$$
m \ddot{u}+c \dot{u}+k u=w(u, \dot{u},\{p\})+r f,
$$

where $u, \dot{u}, \ddot{u}$ are displacement, velocity and acceleration of a body, $w$ is nonlinear force vector, $f$ input excitation vector, $r=$ const, which converts an input excitation vector to the nodal force vector, $\{p\}$ is a vector of model parameters.

The objective is to find $f$ and $\{p\}$ in time interval $[0, T]$, which minimize the target function

$$
J=\varphi\left(u_{T}, \dot{u}_{T}\right)+\int_{0}^{T} \psi(u, \dot{u}, \ddot{u},\{p\}) d t,
$$

where $u, \dot{u}, \ddot{u}$ are displacement, velocity and acceleration at the end of time interval, i.e. when $t=T$. The minimum value of the target function $J$ is ensured when:

$$
\frac{\partial J}{\partial f}=0, \quad \frac{\partial J}{\partial p}=0, \quad \frac{\partial J}{\partial T}=0 .
$$

However, in practice it often appears too difficult to solve the system of nonlinear equations (2.5). Therefore the iterative procedure is applied for minimization of this function, with sensitivity functions

$$
\frac{\partial J}{\partial\{p\}}, \quad \frac{\partial J}{\partial f}, \quad \frac{\partial J}{\partial T}
$$

as the search direction. The procedure for obtaining the sensitivity functions is similar as in [?]. The conjugate variables are used in order to express the variation of the target function in terms of $\{p\}, f(t)$ and $T$. Let us define small variation $\delta f$ of input excitation, $\delta p$ is the variation of parameters, $\delta T$ is the variation of the length of the time interval. As a result, variations $h(t), \dot{h}(t), \ddot{h}(t)$ of displacements, velocities and accelerations of the system will appear.

Now we substitute $f+\delta f,\{p\}+\delta\{p\}, T+\delta T, u+h, \dot{u}+\dot{h}, \ddot{u}+\ddot{h}$ into the target function $J(2.4)$ and obtain the variation of the target function $J$ as: 


$$
\begin{aligned}
\delta J=\frac{\partial \varphi}{\partial u_{T}} \delta u_{T}+\frac{\partial \varphi}{\partial \dot{u}_{T}} \delta & \dot{u}_{T}+\psi\left(u_{T}, \dot{u}_{T}, \ddot{u}_{T},\{p\}\right) \delta T \\
& +\int_{0}^{T}\left(\frac{\partial \psi}{\partial u} h+\frac{\partial \psi}{\partial \dot{u}} \dot{h}+\frac{\partial \psi}{\partial \ddot{u}} \ddot{h}+\frac{\partial \psi}{\partial\{p\}} \delta\{p\}\right) d t .
\end{aligned}
$$

The following transformations are performed in order to express variations $h(t), \dot{h}(t), \ddot{h}(t)$ in terms of variations $\delta f, \delta\{p\}, \delta T$. After multiplication of equation (2.3) by conjugate variables $\lambda(t), \dot{\mu}(t), \ddot{\eta}(t)$ and by performing integration during time interval $[0, T]$ we obtain:

$$
\left\{\begin{array}{l}
\int_{0}^{T}(\lambda m \ddot{u}+\lambda c \dot{u}+\lambda k u) d t=\int_{0}^{T} \lambda w(u, \dot{u}, p) d t+\int_{0}^{T} \lambda(r f) d t, \\
\int_{0}^{T}(\dot{\mu} m \ddot{u}+\dot{\mu} c \dot{u}+\dot{\mu} k u) d t=\int_{0}^{T} \dot{\mu} w(u, \dot{u}, p) d t+\int_{0}^{T} \dot{\mu}(r f) d t \\
\int_{0}^{T}(\ddot{\eta} m \ddot{u}+\ddot{\eta} c \dot{u}+\ddot{\eta} k u) d t=\int_{0}^{T} \ddot{\eta} w(u, \dot{u}, p) d t+\int_{0}^{T} \ddot{\eta}(r f) d t .
\end{array}\right.
$$

After taking variations of both sides of (2.7), the equations read as

$$
\left\{\begin{array}{l}
\lambda_{T}\left(m \ddot{u}_{T}+c \dot{u}_{T}+k u_{T}-w\left(u_{T}, \dot{u}_{T}, p\right)-r f_{T}\right) \delta T \\
\quad+\int_{0}^{T} \lambda(m \ddot{h}+\tilde{c} \dot{h}+\tilde{k} h) d t=\int_{0}^{T} \lambda\left(\frac{\partial w\left(u_{T}, \dot{u}_{T}, p\right)}{\partial p} \delta p+r \delta f\right) d t \\
\dot{\mu}_{T}\left(m \ddot{u}_{T}+c \dot{u}_{T}+k u_{T}-w\left(u_{T}, \dot{u}_{T}, p\right)-r f_{T}\right) \delta T \\
\quad+\int_{0}^{T} \dot{\mu}(m \ddot{h}+\tilde{c} \dot{h}+\tilde{k} h) d t=\int_{0}^{T} \dot{\mu}\left(\frac{\partial w\left(u_{T}, \dot{u}_{T}, p\right)}{\partial p} \delta p+r \delta f\right) d t \\
\ddot{\eta}_{T}\left(m \ddot{u}_{T}+c \dot{u}_{T}+k u_{T}-w\left(u_{T}, \dot{u}_{T}, p\right)-r f_{T}\right) \delta T \\
+\int_{0}^{T} \ddot{\eta}(m \ddot{h}+\tilde{c} \dot{h}+\tilde{k} h) d t=\int_{0}^{T} \ddot{\eta}\left(\frac{\partial w\left(u_{T}, \dot{u}_{T}, \ddot{u}_{T}, p\right)}{\partial p} \delta p+r \delta f\right) d t
\end{array}\right.
$$

where:

$$
\tilde{c}=c-\frac{\partial w(u, \dot{u}, p)}{\partial \dot{u}}, \quad \tilde{k}=k-\frac{\partial w(u, \dot{u}, p)}{\partial u} .
$$

In the case of the variable control time $T$ we may write

$$
\delta u_{T}=h_{T}+\dot{u}_{T} \delta T, \quad \delta \dot{u}_{T}=\dot{h}_{T}+\ddot{u}_{T} \delta T .
$$

Integration by parts, summing and substituting equations (2.9) into (2.8) will result: 


$$
\begin{aligned}
& \left(\lambda_{T}+\dot{\mu}_{T}+\ddot{\eta}_{T}\right)\left(c \dot{u}_{T}+k u_{T}-w\left(u_{T}, \dot{u}_{T}, p\right)-r f_{T}\right) \delta T \\
& \quad-\delta \dot{u}_{T}\left(\dot{\lambda}_{T} m-\dot{\eta}_{T} \tilde{c}_{T}+\eta_{T} \tilde{k}_{T}-\lambda_{T} m-\dot{\mu}_{T} m\right)+\ddot{u}_{T} \delta T\left(\dot{\lambda}_{T} m-\dot{\eta}_{T} \tilde{c}_{T}\right. \\
& \left.+\eta_{T} \tilde{k}_{T}+\ddot{\eta}_{T} m\right)+\delta u_{T}\left(\lambda_{T} \tilde{c}_{T}+\mu_{T} \tilde{k}_{T}+\dot{\eta}_{T} \tilde{k}_{T}\right)-\dot{u}_{T} \delta T\left(\lambda_{T} \tilde{c}_{T}\right. \\
& \left.+\mu_{T} \tilde{k}_{T}+\dot{\eta}_{T} \tilde{k}_{T}\right)+h \int_{0}^{T}(\ddot{\lambda} m-\dot{\lambda} \tilde{c}+\lambda(\tilde{k}-\dot{\tilde{c}})-\mu \dot{\tilde{k}}-\dot{\tilde{k}}) d t \\
& -\dot{h} \int_{0}^{T}(\ddot{\mu} m-\dot{\mu} \tilde{c}+\mu \tilde{k}-\dot{\eta} \dot{\tilde{c}}+\eta \dot{\tilde{k}}) d t+\ddot{h} \int_{0}^{T}(\ddot{\eta} m-\dot{\eta} \tilde{c}+\eta \tilde{k}) d t \\
& =\int_{0}^{T}(\lambda+\dot{\mu}+\ddot{\eta})\left(\frac{\partial w\left(u_{T}, \dot{u}_{T}, \ddot{u}_{T}, p\right)}{\partial p} \delta p+r \delta f\right) d t .
\end{aligned}
$$

Now we require that conjugate variables ensure satisfaction of the following system of equations

$$
\left\{\begin{array}{l}
\ddot{\lambda} m-\dot{\lambda} \tilde{c}+\lambda(\tilde{k}-\dot{\tilde{c}})-\mu \dot{\tilde{k}}-\dot{\eta} \dot{\tilde{k}}=\frac{\partial \psi}{\partial u}, \\
\ddot{\mu} m-\dot{\mu} \tilde{c}+\mu \tilde{k}-\dot{\eta} \dot{\tilde{c}}+\eta \dot{\tilde{k}}=-\frac{\partial \psi}{\partial \dot{u}} \\
\ddot{\eta} m-\dot{\eta} \tilde{c}+\eta \tilde{k}+\eta \dot{\tilde{k}}=\frac{\partial \psi}{\partial \ddot{u}} .
\end{array}\right.
$$

Then the end conditions (at time moment $t=T$ ) are calculated from the equations:

$$
\left\{\begin{array}{l}
\lambda_{T} \tilde{c}_{T}+\mu_{T} \tilde{k}+\dot{\eta}_{T} \tilde{k}_{T}=\frac{\partial \varphi}{\partial u}, \\
\dot{\lambda}_{T} m-\dot{\eta}_{T} \tilde{c}_{T}+\eta_{T} \tilde{k}_{T}-\lambda_{T} m-\dot{\mu}_{T} m=-\frac{\partial \varphi}{\partial \dot{u}_{T}} .
\end{array}\right.
$$

By taking into account that equations (2.12) have more unknown variables than the equations (2.11), the solution of them may be presented as

$$
\lambda_{T}=\dot{\lambda}_{T}=\dot{\mu}_{T}=\dot{\eta}_{T}=0, \quad \mu_{T}=\frac{\partial \varphi}{\partial u_{T}}, \quad \eta_{T}=-\tilde{k}_{T}^{-1} \frac{\partial \varphi}{\partial \dot{u}_{T}} .
$$

Finally equation (2.10) reads as

$$
\begin{array}{r}
\left(\lambda_{T}+\dot{\mu}_{T}+\ddot{\eta}_{T}\right)\left(c \dot{u}_{T}+k u_{T}-w\left(u_{T}, \dot{u}_{T}, p\right)-r f_{T}\right) \delta T+\frac{\partial \varphi}{\partial \dot{u}_{T}} \delta \dot{u}_{T} \\
+\frac{\partial \varphi}{\partial u_{T}} \delta u_{T}+\ddot{u}_{T} \delta T\left(\lambda_{T} m-\dot{\eta}_{T} \tilde{c}_{T}+\eta_{T} \tilde{k}_{T}+\ddot{\eta}_{T} m\right)
\end{array}
$$




$$
\begin{aligned}
& -\dot{u}_{T} \delta T\left(\lambda_{T} \tilde{c}_{T}+\mu_{T} \tilde{k}_{T}+\dot{\eta}_{T} \tilde{k}_{T}\right)+h \int_{0}^{T} \frac{\partial \psi}{\partial u} d t+\dot{h} \int_{0}^{T} \frac{\partial \psi}{\partial \dot{u}} d t \\
& +\ddot{h} \int_{0}^{T} \frac{\partial \psi}{\partial \ddot{u}} d t-\int_{0}^{T}(\lambda+\dot{\mu}+\ddot{\eta})\left(\frac{\partial w\left(u_{T}, \dot{u}_{T}, p\right)}{\partial p} \delta p+r \delta f\right) d t=0 .
\end{aligned}
$$

After entering equation (2.14) into the expression of target function $J$, variation (2.6) reads as:

$$
\delta J=\frac{\partial J}{\partial T} \delta T+\frac{\partial J}{\partial p} \delta p+\int_{0}^{T} \frac{\partial J}{\partial f} \delta f d t,
$$

where sensitivity functions (used as a search direction) are given by:

$$
\begin{aligned}
\frac{\partial J}{\partial f}= & (\lambda+\dot{\mu}+\ddot{\eta}) r, \\
\frac{\partial J}{\partial p}= & \int_{0}^{T}(\lambda+\dot{\mu}+\ddot{\eta})\left(\frac{\partial w\left(u_{T}, \dot{u}_{T}, p\right)}{\partial p} \delta p+\frac{\partial \psi}{\partial\{p\}}\right) d t, \\
\frac{\partial J}{\partial T}= & \psi\left(u_{T}, \dot{u}_{T}, \ddot{u}_{T},\{p\}\right)-\left(\lambda_{T}+\dot{\mu}_{T}+\ddot{\eta}_{T}\right)\left(c \dot{u}_{T}+k u_{T}-w\left(u_{T}, \dot{u}_{T}, p\right)-r f_{T}\right) \\
& -\ddot{u}_{T}\left(\dot{\lambda}_{T} m-\dot{\eta}_{T} \tilde{c}_{T}+\eta_{T} \tilde{k}_{T}+\ddot{\eta}_{T} m\right)+\dot{u}_{T}\left(\lambda_{T} \tilde{c}_{T}+\mu_{T} \tilde{k}_{T}+\dot{\eta}_{T} \tilde{k}_{T}\right) .
\end{aligned}
$$

\subsubsection{Application to CIP model parameter identification problem}

Differential equation of CIP model (2.1) is considered together with the error function (2.2) used as a target function. In our CIP model the control time $T$ and excitation function $f$ are assumed as fixed, so we obtain:

$$
\begin{aligned}
& \partial T=0, \quad \partial f=0, \quad \frac{\partial J}{\partial T}=0, \quad \frac{\partial J}{\partial f}=0, \quad \frac{\partial \psi}{\partial \dot{u}}=0, \\
& \frac{\partial \psi}{\partial u}=\frac{\partial}{\partial u}\left(\frac{1}{2}\left(h u(t)-\frac{I \ddot{u}(t)}{m g}-u_{r e f}(t)\right)^{2}\right)=h\left(h u(t)-\frac{I \ddot{u}(t)}{m g}-u_{r e f}(t)\right), \\
& \frac{\partial \psi}{\partial \ddot{u}}=\frac{\partial}{\partial \ddot{u}}\left(\frac{1}{2}\left(h u(t)-\frac{I \ddot{u}(t)}{m g}-u_{r e f}(t)\right)^{2}\right)=-\frac{I}{m g}\left(h u(t)-\frac{I \ddot{u}(t)}{m g}-u_{r e f}(t)\right) .
\end{aligned}
$$

Equations (2.11) take the form:

$$
\left\{\begin{array}{l}
\ddot{\lambda} m-\dot{\lambda} \tilde{c}+\lambda(\tilde{k}-\dot{\tilde{c}})-\mu \dot{\tilde{k}}-\dot{\eta} \dot{\tilde{k}}=h\left(h u(t)-\frac{I \ddot{u}(t)}{m g}-u_{r e f}(t)\right), \\
\ddot{\mu} m-\dot{\mu} \tilde{c}+\mu \tilde{k}-\dot{\eta} \dot{\tilde{c}}+\eta \dot{\tilde{k}}=0, \\
\ddot{\eta} m-\dot{\eta} \tilde{c}+\eta \tilde{k}=-\frac{I}{m g}\left(h u(t)-\frac{I \ddot{u}(t)}{m g}-u_{r e f}(t)\right),
\end{array}\right.
$$


with initial conditions derived from equations (2.4) and (2.12):

$$
\lambda_{T}=\dot{\lambda}_{T}=\mu_{T}=\dot{\mu}_{T}=\eta_{T}=\dot{\eta}_{T}=0 .
$$

The derivative $\frac{\partial J}{\partial p}$ is calculated from equation (2.16). Since $\frac{\partial \psi}{\partial\{p\}}=0$, we obtain

$$
\frac{\partial J}{\partial p}=\int_{0}^{T}\left((\lambda+\dot{\mu}+\ddot{\eta}) \frac{\partial w\left(u_{T}, \dot{u}_{T}, p\right)}{\partial p}\right) d t,
$$

where $\{p\}=\left\{K_{P}, K_{I}, K_{D}, A, B\right\}$,

$$
w(u, \dot{u},\{p\})=B x(t)-A \dot{y}(t)-\left(K_{P} u+K_{I} \int_{0}^{t} u d t+K_{D} \dot{u}\right) .
$$

Therefore sensitivity function $\frac{\partial J}{\partial p}$ is defined as

$$
\frac{\partial J}{\partial p}=\left\{\begin{array}{l}
\frac{\partial J}{\partial K_{P}} \\
\frac{\partial J}{\partial K_{I}} \\
\frac{\partial J}{\partial K_{D}} \\
\frac{\partial J}{\partial A} \\
\frac{\partial J}{\partial B}
\end{array}\right\}=\left\{\begin{array}{l}
\int_{0}^{T}(-(\lambda(t)+\dot{\mu}(t)+\ddot{\eta}(t)) u(t)) d t, \\
\int_{0}^{T}\left(-(\lambda(t)+\dot{\mu}(t)+\ddot{\eta}(t)) \int_{0}^{t} u(\tau) d \tau\right) d t, \\
\int_{0}^{T}(-(\lambda(t)+\dot{\mu}(t)+\ddot{\eta}(t)) \dot{u}(t)) d t, \\
\int_{0}^{T}(-(\lambda(t)+\dot{\mu}(t)+\ddot{\eta}(t)) \dot{y}(t)) d t, \\
\int_{0}^{T}((\lambda(t)+\dot{\mu}(t)+\ddot{\eta}(t)) x(t)) d t .
\end{array}\right\}
$$

In order to minimize the target function $J$ and solve equation (2.18) the Steepest Descent method was used.

\subsubsection{Implementation}

The CIP model parameter identification algorithm was implemented in Matlab7. White noise time signal used to generate disturbance torque was produced by Matlab function "randn". As reference signal $U_{\text {ref }}$ we used the COP signal recorded during experiments by using sample rate $10 \mathrm{~Hz}$ during $60 \mathrm{~s}$. Body mass and height of COM are measured data of an individual person. We used the mass moment of inertia $I=76 \mathrm{~kg} \cdot \mathrm{m}^{2}$, mass $m=60 \mathrm{~kg}$ and distance of COM from the ankle $h=1.13 \mathrm{~m}$. 


\section{Results of CIP Model Parameter Identification}

The experiments were conducted in order to find the best coincidence of the experimental COP signal (see Figure 3 for signal $U c o p_{r e f}$ ) with the COP signal produced by CIP model (Figure $3, U c o p_{\text {before }}$ is a signal before parameters identification, and $U c o p_{\text {after }}$ a signal after parameters identification). A set of experiments was conducted in order to identify parameters of corrective torque $T_{c}$, keeping the same signal of disturbance torque $T_{d}$. Initial corrective torque and disturbance torque parameters have been set the same as in the experiment described in [?] and commented as being able to produce a realistic COP signal. During experiments the values of $K_{P}, K_{I}$ and $K_{D}$ changed very slightly and the resulting signal $U c o p$ did not changed significantly (see Fig. 3).

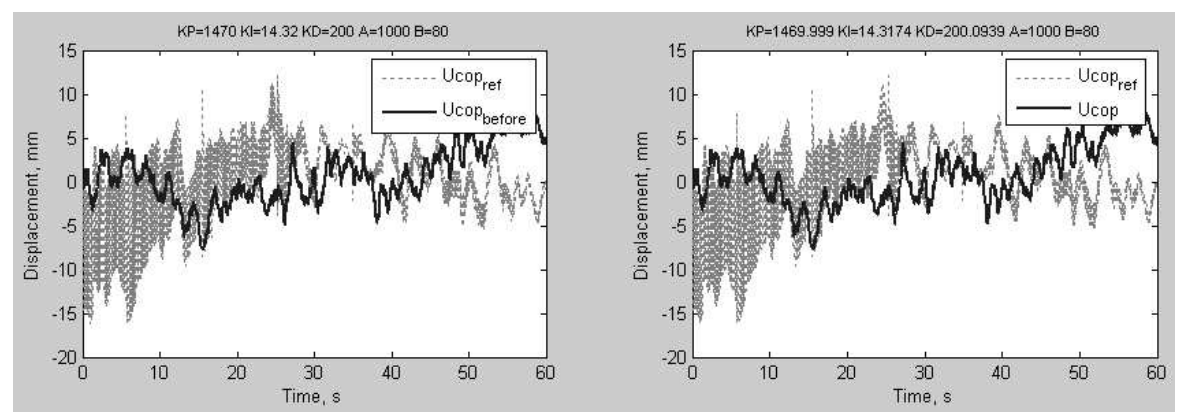

Figure 3. Parameter identification of corrective torque $T_{c}$.

The second set of experiments was conducted in order to investigate the influence of parameters of disturbance torque $T_{d}$ on the CIP model performance. During this experiment the model parameters $K_{P}=1470, K_{I}=$ $14.32, K_{D}=200$ were kept constant, and identification of coefficients $A$ and $B$ performed by means of the error function minimization procedure. As a result, coefficient $A$ decreased from 1000 to 937 while the filter coefficient $B$ increased from 80 to 140 . The absolute value of the error function decreased form $1.277 \mathrm{e}^{-3}$ to $9.8356 \mathrm{e}^{-4}$. In Figure $4 T_{d}$ signal represents CIP model disturbance torque before parameter identification and $T d_{o p t}$ is disturbance with improved values of parameters.

The last set of experiments was conducted in order to identify both excitation (disturbance) parameters $A, B$ and model (control) parameters $K_{P}, K_{I}$ and $K_{D}$. The initial values of model and excitation parameters were set the same as in $R$. J. Peterka's experiment [?] (upper left graph of Figure 5). In upper right graph of Figure 5 the identified CIP model and disturbance torque parameters are shown. $U$ cop represents the COP signal generated by CIP model with identified parameters:

$$
K_{P}=1469, \quad K_{I}=14.3, \quad K_{D}=198, \quad A=1101, \quad B=361 .
$$




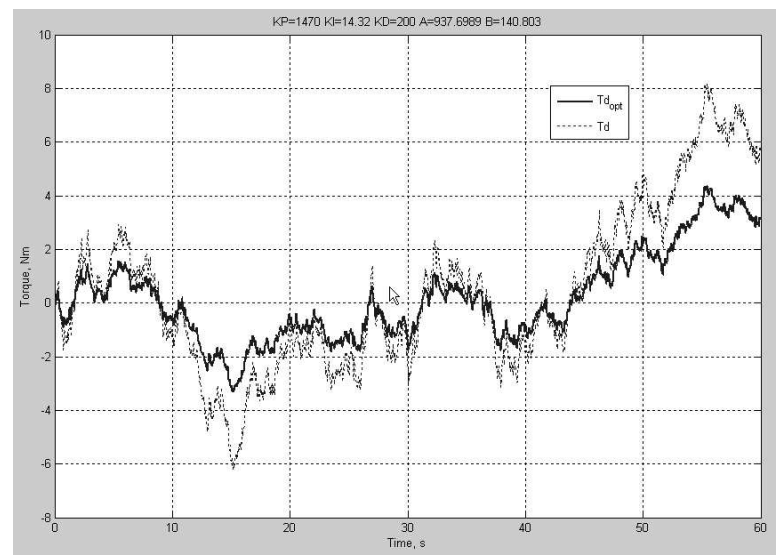

Figure 4. Optimization of disturbance torque $T_{d}$ parameters.
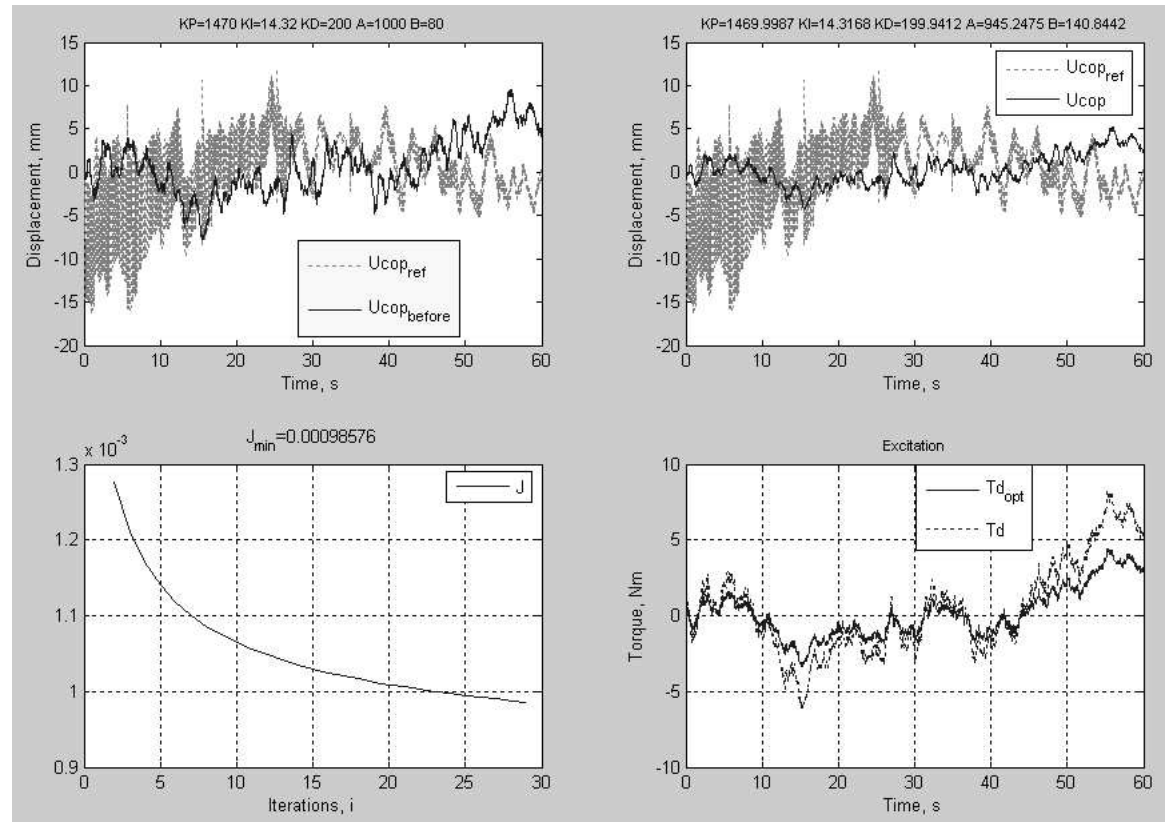

Figure 5. Model and excitation parameter identification.

The absolute value of the target function $J$ decreased form $1.277 \mathrm{e}^{-3}$ to $8.65 \mathrm{e}^{-4}$ (lower left graph of Figure 5). Lower right graph of Figure 5 demonstrates how initial excitation $T_{d}$ was transformed to model identified excitation $T d_{\text {opt }}$.

It can be visually inspected from the Figure 5 that the COP signal of the model (Ucop in top right graph of Figure 5) is highly dependent on disturbance torque $T_{d}\left(T d_{\text {opt }}\right.$ in bottom right graph). 
The experiments revealed that CIP model due to its simplicity was not able to present very high and very low frequency components of COP signal simultaneously: in upper right graph of Figure $5 U c o p_{\text {ref }}$ signal have both high and low frequency components while $U$ cop signal presents only a subset of real COP frequency components.

\section{Conclusions}

The single DOF controlled inverted pendulum human posture model was investigated in order to obtain parameter values which provide satisfactory coincidence between simulation and experimental results. General case of elastic structure optimal design and control method was investigated and extended to the case when the target function is dependent on the displacement, velocity and acceleration of the body. This method was used to identify CIP model parameters (disturbance and control). The error function presenting the cumulative non-coincidence of theoretical and experimentally recorded signal has been minimized with step-by-step procedure, where the gradient of the error function has been used as the search direction. As a result, optimum values of model parameters have been obtained.

The implemented CIP model presented the ability to repeat the recorded benchmark functions with acceptable tolerance. The experiments using real COP signal values revealed that CIP model due to its simplicity was not able to present higher and lower frequency components of COP signal simultaneously. Therefore more realistic structural models which are able to take into account both ankle and hip strategy of human posture should be investigated.

Identified PID regulator parameters $K_{P}, K_{I}, K_{D}$ remained close to their initial values taken from [?] and therefore also may be treated as able to produce a realistic COP signal. While disturbance $T_{d}(t)$ low-pass filter parameters $A$ and $B$ tended to decrease and increase respectively from their initial values, showing that the influence of a random component of disturbance must be smaller and the filter component greater.

The resulting COP signal of the CIP model is highly dependant on disturbance torque $T_{d}$. Method to generate disturbance torque $T_{d}$ dependant on a random signal is not correct when there is an attempt to identify CIP model parameters of a particular experiment. Other means of producing physiologically meaningful disturbance torque must be considered. 


\section{References}

[1] R. Barauskas and R. Krušinskienè. Development and validation of structural models of human posture. In: R. Ciegis(Ed.), CD Proceedings of the 10th International Conference MMA20056CMAM2, June 1-5, 2005, Trakai, Lithuania, $143-150,2005$.

[2] R. Barauskas and V. Ostasevičius. Tampriuju vibrosmūginiu sistemu analize ir optimizavimas. Technologija, Kaunas, 1998.

[3] C.C. Chow and J.J. Collins. Pinned polymer model of posture control. Phys.Rew.E, 52(1), 907 - 912, 1995.

[4] J.J. Collins and C.J. De Luca. Open-loop and closed-loop control of posture: a random-walk analysis of center-of-pressure trajectories. Experimental Brain Research, 95, 308 - 318, 1993.

[5] R. Jacobs. Control model of human stance using fuzzy logic. Biol.Cybern., 63 70, 1997.

[6] P.G. Morasso and M. Schieppati. Can muscle stiffness alone stabilize upright standing? Journal of Neurophysiology, 82, 1622 - 1626, 1999.

[7] R.J. Peterka. Postural control model interpratation of stabilogram diffusion analysis. Biological Cybernetics, 82, $335-343,2000$.

[8] H. Tanaka, M. Nakashizuka, T. Uetake and T. Itoh. The effects of visual input of postural control mechanisms: an analysis of center-of-pressure trajectories using auto regressive model. J.Human Ergol, 29, 15 - 25, 2000. 